\title{
NEAR-INFRARED IMAGING OF PROTO-PLANETARY
}

\section{NEBULAE}

\author{
R.E.S. CLEGG \\ Royal Greenwich Observatory, Madingley Rd, Cambridge CB3 OEZ, UK. \\ and \\ N. A. WALTON and M.J. BARLOW \\ Dept of Physics \& Astronomy, University College London, Gower St, London WC1E \\ $6 B T, U K$.
}

It is not really known how low and intermediate mass stars eject mass to form PNs. We present preliminary results from a programme of near-IR imaging, in which we study a sequence of objects, from extreme AGB stars through protoplanetaries to young, compact PNs. We airn to study the sequence of morphologies, to see where the onset of bipolar shaping occurs, and to use the IR molecular hydrogen lines to map neutral regions around ionized nebulae.

The observations are from the $4.2 \mathrm{~m}$ William Herschel Telescope at La Palma, with the near-IR camera 'FAST' developed by Prof. Genzel's group at MPE, Garching. A CVF and a Fabry-Perot together provide a 'tunable filter' with resolving power of 900 . Observations were taken in $\mathrm{H} \mathrm{I}$ Brackett $\gamma$, the $\mathrm{H}_{2} v=1-0$ and 2-1 $\mathrm{S}(1)$ lines, He II $n=6-4$, [Si VI] and [Si VII] lines, all in the ' $\mathrm{K}$ ' band window.

We present preliminary results for four objects. For NGC 7027, the $\mathrm{H}_{2} \mathrm{v}=1-0$ S(1) map shows a remarkable set of 4 'loops' outside the bright ionised ring. (The ring itself is completely absent from the $\mathrm{H}_{2}$ maps). This $\mathrm{H}_{2}$ emission is mostly collisionally-excited, and it traces warm, dense portions of the neutral shell. The hydrodynamical model predictions of Mellema et al. (A \& Ap., 1992) for two-wind, bipolar structures with exit shocks may explain the 4 loops. A further map in the $v=2-1$ line will trace the relative distributions of collisionally-excited and UVexcited $\mathrm{H}_{2}$.

CRL 2688 shows two symmetric pairs of $\mathrm{H}_{2}$ emission knots, and our new deep maps reveal loops of emission joining the pairs. Comparison of the $v=1-0$ and $2-1$ $\mathrm{S}(1)$ line maps indicates a gas kinetic temperature of about $1200 \mathrm{~K}$. This must refer to shocked gas, since there is no UV source in this object.

For $\mathrm{BD}+30^{\circ} 3639$ our $\mathrm{H}_{2}$ line map shows a $15 \times 10^{\prime \prime}$ elliptical 'neutral' nebula around a compact, 7" diameter spherical ionized nebula. The $\mathrm{H}_{2}$ emission is however extremely non-uniform. Since the $\mathrm{H}_{2}$ line is probably excited by UV photons, it is suggested that dust clouds between the central star and the outer parts of the large elliptical shell provide greatly-varying extinction in the UV.

Our images of the compact $\mathrm{PN}$ Vy 2-2 to have extended emission in the $\mathrm{H}_{2}$ $v=1-0 \mathrm{~S}(1)$ line, at PA about $30 \mathrm{deg} \mathrm{E}$ of $\mathrm{N}$. This corresponds to an extension seen in the $4.9 \mathrm{GHz}$ VLA map of Seaquist \& Davis (1983), and suggests that the emission may be shock-excited along two polar flows, as is the case for the two main lobes in CRL 2688. 